Alice Helena de Resende Nóra Pacheco'

Daniele Marano Rocha Araujo'

ELISA MARIa de AQUinO LACERDA²

GILBERTO KAC

\title{
Consumo de cafeína por grávidas usuárias de uma Unidade Básica de Saúde no município do Rio de Janeiro
}

\author{
Caffeine consumption by pregnant women selected from a Health Care \\ Center in the municipality of Rio de Janeiro, Brazil
}

Palavras-chave

Cafeína

Gravidez

Consumo de alimentos

Menarca

Preparações farmacêuticas

Keywords

Caffeine

Pregnancy

Food consumption

Menarche

Medicines

\section{Resumo}

OBJETIVO: avaliar o consumo de cafeína em gestantes e sua associação com variáveis demográficas, socioeconômicas, reprodutivas e comportamentais e com o estado nutricional materno. MÉTODOS: trata-se de estudo do tipo transversal, realizado entre 2005 e 2007. A presente análise refere-se ao período entre a oitava e a $13^{a}$ semana gestacional, sendo realizada com 255 gestantes entre 18 e 40 anos, usuárias de uma Unidade Básica de Saúde no município do Rio de Janeiro, Rio de Janeiro. A variável "desfecho" foi o consumo de cafeína quantificado por meio de questionário de freqüência alimentar semiquantitativo, previamente validado, o qual continha uma lista de alimentos com 81 itens e oito opções de freqüência de consumo. A ingestão de cafeína foi quantificada a partir do consumo de: chocolate em pó/Nescau ${ }^{\circledR}$, chocolate em barra ou bombom, refrigerante, café e mate. A análise estatística foi realizada por meio de modelo hierarquizado de regressão linear múltipla. RESULTADOS: a mediana e o consumo médio de cafeína foram, respectivamente, de 97,5 e 121,1 mg (desvio padrão, dp=128,4). Já o consumo elevado da substância (>300 mg/dia) foi observado em $8,3 \%$ das gestantes. No modelo multivariado, observou-se que mulheres cuja menarca ocorreu mais cedo $(\beta=-0,15)$, com maior número de pessoas vivendo na casa $(\beta=0,17)$ e que não faziam uso de medicamentos $(\beta=-0,24)$ apresentaram maior tendência ao consumo elevado de cafeína e esta foi estatisticamente significativa $(p<0,05)$. CONCLUSÕES: o consumo de cafeína pela maioria das gestantes foi inferior ao limite de $300 \mathrm{mg} /$ dia preconizado em outros estudos. Observou-se tendência ao consumo elevado de cafeína nas gestantes cuja menarca ocorreu mais cedo, com maior número de pessoas vivendo na casa e que não faziam uso de medicamentos.

Abstract

PURPOSE: to determine caffeine consumption in pregnant women and to evaluate its association with demographic, socioeconomic, reproductive, lifestyle and maternal nutritional status. METHODS: it is a cross-sectional study performed between 2005 and 2007. The present analysis refers to the period among the 8th and 13th gestational week and included 255 pregnant women from 18 to 40 years, clients of a municipal health center in Rio de Janeiro. The outcome variable was caffeine consumption, quantified by a semi-quantitative food frequency questionnaire, which count with a list containing 81 items and eight options of consumption frequencies; besides it being previously validated in a sample of employees of the State University of Rio de Janeiro. The caffeine intake was quantified starting from the consumption of: powdered chocolate, chocolate bar or chocolate, soft drink, coffee and mate tea. The statistical analysis was performed by means of fitting a multivariate linear regression. RESULTS: the median and the mean caffeine consumption were, respectively, 97.5 and $121.1 \mathrm{mg}$ (standard deviation, $\mathrm{sd}=128.4$ ). The high caffeine consumption (> $300 \mathrm{mg} /$ day) was observed in $8.3 \%$ of pregnant women. It was observed in the multivariate model that women with earlier menarche $(\beta=-0.15)$, with more household partners $(\beta=0.17)$ and who didn't make use of medicines $(\beta=-0.24)$ presented larger tendency to high caffeine consumption association that was statistically significant ( $p<0.05$ ). CONCLUSIONS: the caffeine consumption for most of the pregnant women was inferior to the limit of $300 \mathrm{mg} /$ day as commited in other studies. Tendency was observed toward higher consumption of caffeine in pregnant women with earlier menarche, with more household partners and who didn't make use of medicines.

Correspondência:

Alice Helena de Resende Nóra Pacheco Departamento de Nutricão Social e Aplicada do Instituto de Nutriç̄ôo Josué de Castro da Universidade Federal do Rio de Janeiro Avenida Brigadeiro Trompowski, $s / n, C C S$, bloco $J, 2^{\circ}$ andar, sala 29 - Cidade Universitária, llha do Fundão (EP: 21941-590 - Rio de Janeiro/RJ Fone: (21) 2562-6595 E-mail: aliceheleno@ufri.br
Instituto de Nutrição Josué de Castro da Universidade Federal do Rio de Janeiro - UFRJ - Rio de Janeiro (RJ), Brasil. Apoio: Conselho Nacional de Desenvolvimento Científico e Tecnológico - CNPq - e Coordenação de Aperfeiçoamento de Pessoal de Nível Superior - Capes.

'Pós-graduandos pelo Programa de Pós-Graduação em Nutrição do Instituto de Nutrição Josué de Castro da Universidade Federal do Rio de Janeiro - UFRJ - Rio de Janeiro (RI), Brasil.

2 Professor Adjunto do Instituto de Nutrição Josué de Castro da Universidade Federal do Rio de Janeiro - UFRJ - Rio de Janeiro (RJ), Brasil.

${ }^{3}$ Doutor, Professor Adjunto do Instituto de Nutrição Josué de Castro da Universidade Federal do Rio de Janeiro - UFRJ - Rio de Janeiro (RJ); Pesquisador com Bolsa de Produtividade do Conselho Nacional de Desenvolvimento Científico e Tecnológico - CNPq. 


\section{Introdução}

O consumo de cafeína é bastante freqüente em todo o mundo ${ }^{1}$. Alguns estudos demonstram que, durante a gestação, ocorre aversão aos produtos cafeinados no primeiro trimestre, particularmente ao café, levando à interrupção ou redução no consumo ${ }^{2}$. Em estudo com dados do Reino Unido, Dinamarca e Austrália, sobre o consumo de alimentos fontes de cafeína, foi observada redução de 20 a 22\% na ingestão da substância entre as gestantes da amostra representativa da população do Reino Unido, quando comparadas a outros grupos na população geral daquele país ${ }^{3}$. Na avaliação das mudanças dietéticas entre o primeiro e o segundo trimestres de gestação, em gestantes americanas com alto nível de escolaridade e renda, tal redução foi de, aproximadamente, $30 \%$ na ingestão de cafeína pelas gestantes ${ }^{4}$.

Apesar deste possível comportamento de aversão à cafeína ou redução do consumo, alguns estudos têm demonstrado a associação entre a ingestão de produtos cafeinados e efeitos não desejáveis para o feto, tais como baixo peso ao nascer, aborto espontâneo, retardo de crescimento uterino e aumento do risco de ruptura precoce das membranas ${ }^{5,6}$, acarretando aumento das taxas de morbidade e mortalidade perinatal e neonatal ${ }^{7}$. Esses desfechos perinatais indesejáveis são devidos, sobretudo, à ausência, no feto, de enzimas necessárias para a demetilação da cafeína ${ }^{8}$, deixando o mesmo exposto por um longo período da vida intra-uterina a esta substância ${ }^{2,9}$. Alguns estudos relatam que, durante a gestação, a cafeína aumenta os níveis de catecolaminas no sangue e esses hormônios acarretam efeitos negativos para o feto, devido à vasoconstrição e à hipóxia fetal ${ }^{10}$.

Quanto ao nível de ingestão observado, o valor mínimo de cafeína consumido e considerado como risco foi $\geq 71 \mathrm{mg} / \mathrm{dia}^{5}$ e o valor máximo, $>571 \mathrm{mg} / \mathrm{dia}^{6}$. Atualmente, as evidências disponíveis sugerem ser prudente que mulheres grávidas limitem o consumo de cafeína a $300 \mathrm{mg} / \mathrm{dia}$, para reduzir a probabilidade de aborto espontâneo ou crescimento fetal prejudicado ${ }^{11}$. No entanto, mulheres que ingerem quantidades elevadas de cafeína consomem mais álcool, fumam em quantidade superior e apresentam baixo nível educacional ${ }^{10}$. Ademais, o hábito de tomar café, comparativamente ao consumo de chá, em uma amostra populacional de indivíduos de ambos os sexos, entre 25 e 64 anos de idade, esteve associado a um estilo de vida menos saudável, em estudo realizado em Viena, Áustria ${ }^{12}$.

De modo geral, os autores têm buscado, primordialmente, apontar as conseqüências para o concepto do consumo elevado de cafeína durante a gestação. No entanto, poucos são os que têm atentado para os possíveis fatores socioeconômicos, obstétricos, nutricionais e comportamentais que podem estar envolvidos na ingestão dessa substância e caracterizar o consumo elevado ou não de cafeína no período da gravidez. Sendo assim, o presente estudo teve como objetivo investigar os fatores associados ao consumo de cafeína com base em dados de uma coorte de gestantes do município do Rio de Janeiro, Rio de Janeiro, na tentativa de obter informações adequadas que direcionem a orientação a ser dada a essas gestantes.

\section{Métodos}

Trata-se de estudo com desenho do tipo transversal realizado no período de junho de 2005 a abril de 2007 e que contou com entrevistas e monitoramento do estado nutricional materno entre a oitava e a $13^{\mathrm{a}}$ semana gestacional.

O estudo foi realizado no Posto de Saúde Madre Tereza de Calcutá (PSMTC), localizado na Ilha do Governador, no município do Rio de Janeiro. O posto é situado no bairro Bancários e atende a uma demanda local de classe socioeconômica menos favorecida.

As gestantes foram recrutadas no serviço de pré-natal do PSMTC e convidadas a participar do estudo quando procuraram atendimento na unidade de pré-natal pela primeira vez. Os critérios de elegibilidade para a entrada no estudo foram: estar entre a oitava e $13^{\text {a }}$ semana de gestação, ter entre 18 e 40 anos de idade, não apresentar gestação gemelar, não apresentar doenças crônicas não transmissíveis nem doenças infecto-parasitárias e residir na área programática do estudo.

Inicialmente, foram selecionadas 348 gestantes, sendo 292 elegíveis, ou seja, as outras 56 gestantes não atenderam aos critérios de elegibilidade acima. Dentre as 292 elegíveis, 37 recusaram participar da pesquisa ou faltaram à primeira entrevista, contabilizando 12,6\% de perdas. Assim, ao final, 255 mulheres, entre as que atenderam aos critérios de elegibilidade e aceitaram participar do estudo, foram entrevistadas.

No entanto, tendo em vista que três destas 255 mulheres apresentaram um consumo de cafeína - sobretudo de café - muito acima do esperado ( $>800 \mathrm{mg} / \mathrm{dia}$ ou $+3 \mathrm{dp}$ ), elas foram excluídas das demais análises, sendo consideradas outliers. Assim, o estudo incluiu 252 gestantes para efeito das análises das médias de consumo de cafeína por variáveis categóricas e da regressão linear (variável contínua) bivariada e múltipla.

A variável "desfecho" do estudo foi o consumo de cafeína na gestação. Foi utilizado um questionário de freqüência de consumo alimentar (QFCA) semiquantitativo, o qual continha uma lista de alimentos contendo 81 itens e oito opções de freqüência de consumo nos últimos meses, isto é, desde que a mulher descobriu a gravidez até a data da entrevista. Este QFCA foi previamente validado em uma 
amostra de funcionários da Universidade Estadual do Rio de Janeiro (UERJ) $)^{13}$, porém não em gestantes.

A ingestão de cafeína foi quantificada a partir do consumo de chocolate em pó/Nescau ${ }^{\circledR}$ (colher de sopa, $16 \mathrm{~g})^{14}$, chocolate em barra ou bombom (30 g ou dois bombons) $)^{14}$, refrigerante (copo duplo, $\left.240 \mathrm{~mL}\right)^{14}$, café (xícara, $180 \mathrm{~mL})^{14}$ e mate (copo duplo, $\left.240 \mathrm{~mL}\right)^{14}$, distribuídos em oito possibilidades de freqüência: mais de três vezes por dia (3x/dia), 2-3x/dia, 1x/dia, 5-6x/semana, 2-4x/semana, $1 \mathrm{x} /$ semana, $1-3 \mathrm{x} /$ mês e nunca/quase nunca. Tais freqüências foram transformadas em freqüências diárias e essas foram multiplicadas pelas quantidades consumidas dos alimentos, conforme o teor de cafeína de cada produto, para que, finalmente, fossem obtidas as quantidades diárias transformadas em miligramas de cafeína.

Para esta conversão, as seguintes referências foram adotadas: USDA ${ }^{15}$, para quantificar o conteúdo de cafeína do achocolatado em pó; $\mathrm{WHO}^{16}$ e Santos et al. ${ }^{17}$, para o chocolate em barra e/ou bombom; $\mathrm{WHO}^{16}$, Santos et al. ${ }^{17}$ e USDA ${ }^{15}$, para o refrigerante; Camargo e Toledo ${ }^{18}$, para o café; e Santos et al. ${ }^{17}$, para o teor de cafeína no mate. Desse modo, a quantidade ingerida de alimentos foi convertida para miligramas $(\mathrm{mg})$ de cafeína. No presente estudo, não foi quantificado o teor de cafeína presente no chá ou nos medicamentos utilizados, produtos esses que não fizeram parte do QFCA.

Sabe-se que alguns produtos modificados, como alimentos e bebidas light ou diet, possuem mais cafeína que suas respectivas versões tradicionais (por exemplo, o chocolate meio amargo possui mais cafeína que o chocolate ao leite; a Coca-cola ${ }^{\circledR}$ light possui mais cafeína que a tradicional). No intuito de minimizar esse viés no cálculo da cafeína consumida pelas gestantes, foram calculadas e utilizadas as médias dos teores de cafeína encontrados nestes diferentes produtos.

Calculou-se a quantidade de cafeína ingerida por dia e por fonte e somaram-se todas as fontes de cafeína que a gestante utilizava, obtendo-se o consumo médio diário. Verificou-se a freqüência de gestantes com consumo de cafeína superior a $300 \mathrm{mg} / \mathrm{dia}$, considerado elevado de acordo com diferentes estudos ${ }^{1,9,11,17}$. Para efeito das análises, optou-se por utilizar a variável em sua forma contínua.

A partir do elenco de variáveis coletadas na pesquisa, foi construído um modelo hierarquizado para análise estatística das possíveis variáveis associadas ao consumo de cafeína na gestação, por níveis de associação. As seguintes co-variáveis foram incluídas no modelo e nas análises, conforme os seguintes níveis de determinação e categorias: bloco de variáveis demográficas e socioeconômicas: idade ( $<25, \geq 25$ anos); cor da pele segundo observação do entrevistador (branca, parda e preta); idade na menarca $(<12,12$ a $15,>15$ anos); renda familiar per capita ( $<1,1$ a 2, $>2$ salários mínimos); escolaridade ( $\leq 8,9$ a $11, \geq 12$ anos de estudo); número de pessoas que vivem na casa ( 1 a $2,3, \geq 4$ pessoas); situação conjugal (casada, em união, não vive em união) e trabalho fora de casa (sim, não). O bloco de variáveis reprodutivas incluiu: paridade ( $\leq 2,>2$ gestações); história prévia de aborto espontâneo (sim, não); história prévia de baixo peso ao nascer (sim, não) e história prévia de prematuridade (sim, não). As variáveis comportamentais incluíram prática de atividade física (sim, não); uso de contraceptivos orais (sim, não); uso de medicamentos (sim, não); hábito de fumar (sim, não); número de cigarros ( 1 a 9,10 a 19, $\geq 20$ cigarros); idade em que começou a fumar ( $<15,15$ a 20 e $>20$ anos); tempo de tabagismo ( $<2,2$ a 5, $\geq 6$ anos); uso de álcool (sim, não); idade em que começou a beber $(<15,15$ a 20 e $>20$ anos) e tempo de etilismo ( $\leq 3,4$ a $8,>8$ anos).

Objetivando maximizar a qualidade das informações coletadas, os entrevistadores foram treinados para a aplicação dos questionários e as medidas antropométricas foram padronizadas, utilizando-se o protocolo de Lohman et al. ${ }^{19}$. A coleta de sangue para avaliação de anemia foi realizada por profissional destinado para essa finalidade, da própria Unidade Básica de Saúde, e analisada em laboratório filiado ao Sistema Único de Saúde (SUS) na Ilha do Governador. Um estudo piloto foi realizado para testar os instrumentos, a coleta de dados e a logística do estudo.

\section{Análise estatística}

O banco de dados foi construído por dupla digitação, seguida da análise de consistência dos dados utilizando-se o software Epi-Info, versão 6.02. Procedeu-se, então, à análise descritiva das principais variáveis com o intuito de determinar as características da amostra e o consumo de cafeína - geral e por alimentos consumidos.

A distribuição de cada co-variável da amostra foi realizada com o objetivo de definir as melhores categorias para análises, as quais foram distribuídas conforme os blocos de variáveis descritas anteriormente. Utilizou-se o teste do $\chi^{2}$ para comparar proporções e verificar possível correlação entre as variáveis de exposição e o consumo de cafeína abaixo ou acima de $300 \mathrm{mg} /$ dia pelas gestantes. O teste exato de Fisher foi aplicado nos casos em que uma das células $2 \times 2$ era menor que cinco. A comparação das médias de consumo de cafeína por categorias das variáveis explicativas foi realizada por meio da diferença de médias com respectivos intervalos de confiança de 95\% (IC95\%) empregando o teste $t$ de Student, para as variáveis dicotômicas, e a análise de variância (ANOVA), para as com três ou mais categorias.

Considerando-se que a variável "consumo de cafeína" não tinha distribuição normal, foi utilizado o logaritmo 
desta variável para as análises de regressão. Foram realizadas análises bivariadas para avaliação das associações brutas das variáveis explanatórias com o consumo de cafeína, utilizando-se como medida de efeito o coeficiente de regressão linear bruto (ß), com IC95\%. Também se utilizou essa estratégia de análise para determinar os possíveis fatores de confusão.

Posteriormente, foi realizada análise multivariada, do tipo regressão linear múltipla, utilizando o modelo hierarquizado em três níveis de associação. Essa abordagem definiu a ordem de entrada das variáveis no modelo de regressão. Primeiro, as variáveis do nível um foram ajustadas entre si. Após, entraram na regressão as variáveis do nível dois, sendo ajustadas entre si e para as do nível anterior. Nesta análise, utilizou-se, como medida de efeito, o coeficiente de regressão linear ajustado $\left(\beta_{A J}\right)$, com IC95\%. Na análise múltipla, foram mantidas, para controle de confundimento, as variáveis que se associaram com o desfecho a um $\mathrm{p}<0,30^{20}$ na análise bivariada, e foram consideradas associações significativas com o consumo de cafeína aquelas com $\mathrm{p}<0,05$. Todas as análises foram realizadas no pacote estatístico Statistical Package for Social Sciences ${ }^{\circledR}$ (SPSS) 13.0.

$\mathrm{O}$ estudo de coorte que originou este trabalho foi aprovado pelo Comitê de Ética em Pesquisa da Secretaria Municipal de Saúde do Rio de Janeiro (SMS/RJ) e está de acordo com os princípios éticos de não maleficência, beneficência, justiça e autonomia, contidos na resolução 196/96, do Conselho Nacional de Saúde (CNS) ${ }^{21}$. A participação na pesquisa foi condicionada à assinatura de um termo de consentimento livre e esclarecido por parte dos avaliados.

\section{Resultados}

Observou-se que $96 \%$ das mulheres consumiram algum alimento fonte de cafeína durante o primeiro trimestre da gravidez. Dessas mulheres, apenas 8,3\% apresentaram consumo elevado, ou seja, superior a $300 \mathrm{mg} /$ dia de cafeína. Três gestantes $(1,2 \%)$, por apresentarem consumo de cafeína - sobretudo de café, muito acima do esperado (superior a $800 \mathrm{mg} / \mathrm{dia}$ ou $+3 \mathrm{dp}$ ), foram excluídas das análises.

O produto fonte de cafeína menos consumido pelas gestantes foi o mate, com uma prevalência de 9,1\% e contribuindo com apenas $2,8 \%(3,4 \mathrm{mg})$ do consumo total de cafeína. $\mathrm{O}$ produto cafeinado mais consumido foi o refrigerante $(75,4 \%)$ e o que mais contribuiu para o consumo total de cafeína foi o café, sendo responsável por $67,8 \%$ (82,1 mg) da cafeína consumida (Tabela 1).

A maioria das gestantes estudadas tinha menos de 25 anos $(56,3 \%)$, era parda $(64,9 \%)$, teve sua menarca entre 12 e 15 anos de idade $(69,1 \%)$, apresentava renda per capita inferior a um salário mínimo $(61,2 \%)$ e tinha menos de 11 anos de estudo (71,9\%, conforme Tabela 2$)$, vivia em união $(55,8 \%)$ e trabalhava fora de casa $(57,1 \%)$. No que diz respeito ao consumo de bebidas alcoólicas, $12,3 \%$ eram etilistas. Em relação ao estado nutricional materno, $18,4 \%$ apresentaram baixo peso pré-gestacional e $15,3 \%$ apresentaram obesidade pré-gestacional (categorias não demonstradas na tabela). A presença de anemia foi observada em 5,8\% das gestantes (dados não apresentados em tabela).

Em relação ao consumo de cafeína, as maiores médias foram observadas em gestantes com escolaridade entre nove e 11 anos de estudo $(\mathrm{p}=0,06)$, não usuárias de medicamentos ( $\mathrm{p}=0,002)$, fumantes $(\mathrm{p}=0,03)$, que fumavam 20 ou mais cigarros por dia $(\mathrm{p}=0,01)$, que começaram a fumar com menos de 15 anos de idade $(\mathrm{p}=0,04)$, com seis ou mais anos de tabagismo $(\mathrm{p}=0,08)$ e usuárias de bebidas alcoólicas $(\mathrm{p}=0,07)$, de acordo com a Tabela 2 .

Não se verificou diferença significativa na proporção de gestantes com consumo elevado de cafeína (>300 mg/dia), segundo as variáveis: idade, uso de medicamentos, idade na menarca, história prévia de prematuridade, índice de massa corpórea (IMC) pré-gestacional e presença de anemia. Contudo, este hábito foi mais freqüente $(\mathrm{p}=0,05)$ entre as gestantes tabagistas $(17,9 \%)$ do que entre as não tabagistas $(7,1 \%)$ e apresentou uma tendência a ser também mais freqüente $(\mathrm{p}=0,08)$ entre as mulheres com mais que duas gestações $(13,2 \%)$ contra as com paridade menor ou igual a duas $(6,5 \%)$, conforme Tabela 3.

Tabela 1 - Proporção de gestantes consumidoras de cafeína, média de consumo e contribuição da cafeína ingerida, a partir de cada produto, em relação ao consumo total da substância em gestantes usuárias de uma Unidade Básica de Saúde na llha do Governador, Rio de Janeiro

\begin{tabular}{|c|c|c|c|c|c|}
\hline \multirow{2}{*}{ Produto cafeinado } & \multicolumn{2}{|c|}{ Consumidoras* } & \multirow{2}{*}{$\begin{array}{c}\text { Quantidade de cafeína no } \\
\text { produto }\end{array}$} & \multirow{2}{*}{$\begin{array}{c}\text { Média (dp) } \\
\text { (mg/dia) de cafeína }\end{array}$} & \multirow{2}{*}{$\begin{array}{l}\text { Contribuição do produto no } \\
\text { consumo total de cafeína (\%) }\end{array}$} \\
\hline & $n$ & $\%$ & & & \\
\hline Chocolate & 102 & 40,5 & $66,6 \mathrm{mg} / 100 \mathrm{~g}$ & $7,04(31,0)$ & 5,8 \\
\hline Refrigerante & 190 & 75,4 & $10 \mathrm{mg} / 100 \mathrm{~mL}$ & $24,8(55,3)$ & 20,5 \\
\hline Café & 153 & 60,7 & $54,2 \mathrm{mg} / 100 \mathrm{~mL}$ & $82,1(108,3)$ & 67,8 \\
\hline
\end{tabular}

*Amostra corresponde ao número total de mulheres no estudo $(\mathrm{n}=255)$, excluindo-se as gestantes com consumo acima de $800 \mathrm{mg} / \mathrm{dia}(\mathrm{n}=3)$; total=252. 
Tabela 2 - Médias e intervalo de confiança (IC95\%) do consumo de cafeína (mg) em gestantes usuárias de uma Unidade Básica de Saúde na Ilha do Governador, segundo variáveis explicativas

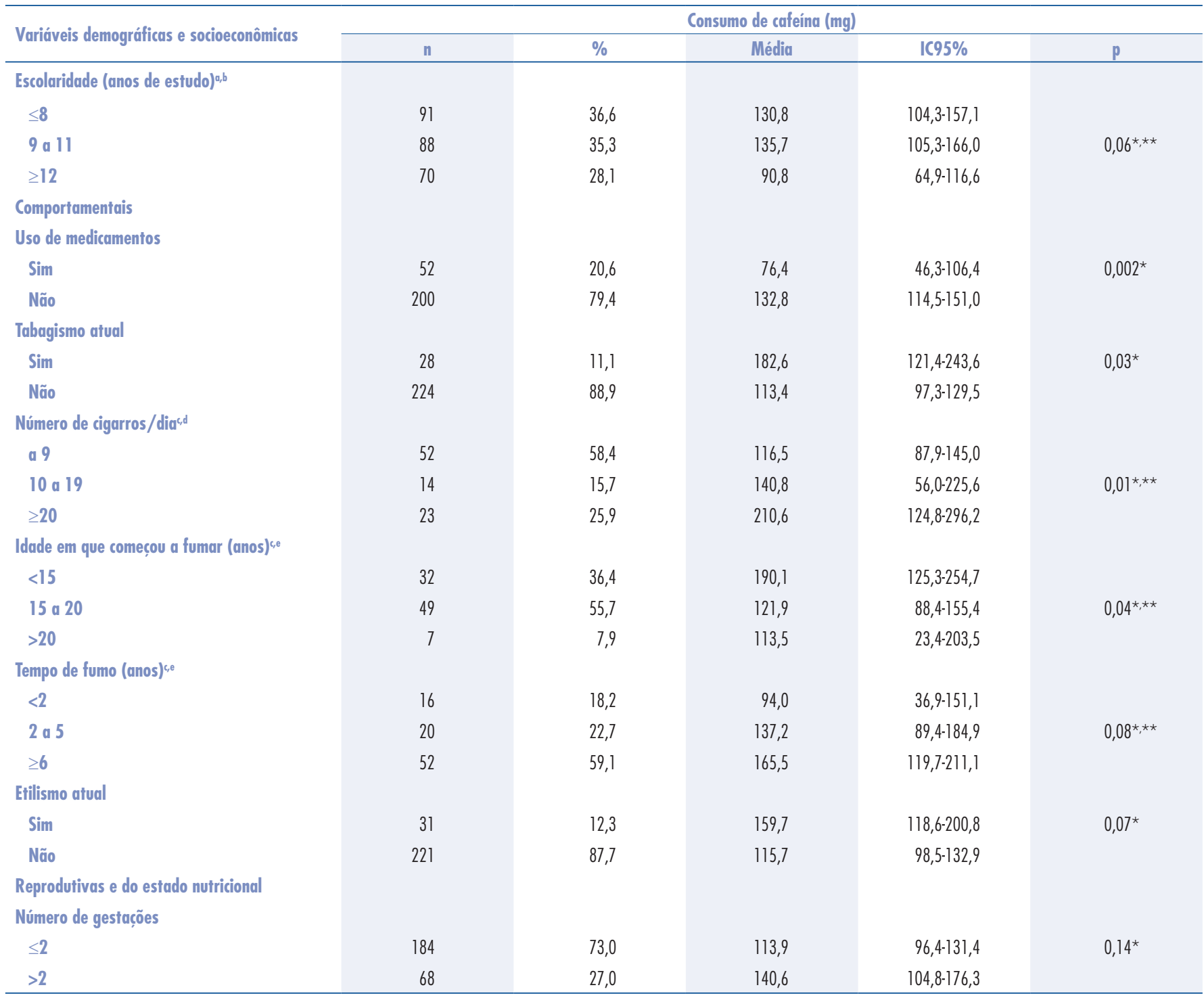

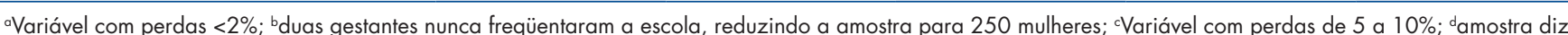
respeito às 29 gestantes que fumam somadas às 64 que já fumaram $(n=93) ;{ }^{e}=$ amostra diz respeito a fumantes atuais e ex-fumantes.

"Teste $t$ de Student para variáveis dicotômicas e ANOVA para as com três ou mais categorias; " valor de p para tendência linear.

Tabela 3 - Prevalência do consumo de cafeína segundo categorias de consumo na amostra de gestantes usuárias de uma Unidade Básica de Saúde na Ilha do Governador, Rio de Janeiro

\begin{tabular}{|c|c|c|c|c|c|c|c|}
\hline \multirow{3}{*}{ Variáveis } & \multicolumn{7}{|c|}{ Consumo de cafeína (mg) } \\
\hline & \multicolumn{2}{|c|}{$\leq 300 \mathrm{mg} / \mathrm{dia}$} & \multicolumn{2}{|c|}{$>300$ mg/dia } & \multicolumn{2}{|c|}{ Total } & \multirow{2}{*}{$\begin{array}{l}\chi^{2} \\
p\end{array}$} \\
\hline & n & $\%$ & n & $\%$ & n & $\%$ & \\
\hline \multicolumn{8}{|c|}{ Tabagismo afual } \\
\hline Não & 208 & 92,9 & 16 & 7,1 & 224 & 100 & \\
\hline \multicolumn{8}{|c|}{ Número de gestações } \\
\hline \multicolumn{8}{|c|}{ Prematuridade préviaa, ${ }^{a} b$} \\
\hline Sim & 16 & 84,2 & 3 & 15,8 & 19 & 100 & $0,21^{*}$ \\
\hline Não & 121 & 92,4 & 10 & 7,6 & 131 & 100 & \\
\hline
\end{tabular}

QVariável com perdas inferiores a $2 \%$; ${ }^{b}$ refere-se às 152 gestantes que não estavam grávidas pela primeira vez.

*Teste exato de Fisher. 
Tabela 4 - Regressão linear bivariada para consumo de cafeína (mg) em gestantes usuárias de uma Unidade Básica de Saúde na Ilha do Governador, Rio de Janeiro

\begin{tabular}{lccc}
\hline \multirow{2}{*}{ Variáveis } & \multicolumn{3}{c}{ Consumo de cafeína (mg) } \\
\cline { 2 - 4 } & $\beta^{\mathrm{b}}$ & \multicolumn{1}{c}{$I \mathrm{C} 95 \%$} & $\mathrm{p}^{\mathrm{c}}$ \\
\hline Idade na menarca (anos) & $-0,02$ & $-0,076-0,018$ & 0,22 \\
Número de pessoas que vivem na casa & 0,04 & $-0,009-0,095$ & 0,10 \\
História prévia de baixo peso ao nascer (não/sim)* & 0,18 & $-0,129-0,506$ & 0,24 \\
Hisłória prévia de prematuridade (não/sim)* & 0,24 & $-0,060-0,543$ & 0,11 \\
Uso de medicamentos (não/sim)* & $-0,24$ & $-0,438-0,046$ & 0,01 \\
Tabagismo afual (não/sim)* & 0,24 & $0,116-0,555$ & 0,05 \\
Idade em que começou a fumar (annos) & $-0,02$ & $-0,057-0,015$ & 0,24 \\
Tempo de fumo (anos) & 0,01 & $-0,005-0,037$ & 0,13 \\
Etilismo afual (não/sim)* & 0,22 & $-0,018-0,467$ & 0,06 \\
Níveis de hemoglobina (mg/dL) & $-0,03$ & $-0,103-0,027$ & 0,24 \\
\hline
\end{tabular}

aLog do consumo de cafeína; ${ }^{b} \beta=$ coeficiente de regressão linear; 'valor de $p$ da regressão linear.

*Variáveis dicotômicas para as quais a primeira categoria é a de referência e a segunda é de risco.

$\mathrm{Na}$ regressão linear bivariada, as seguintes variáveis mostraram-se associadas ao consumo de cafeína: idade na menarca $(\beta=-0,02)$, número de pessoas que vivem na casa $(\beta=0,04)$, baixo peso ao nascer prévio [não (referência)/ sim (risco), $\beta=0,18$ ], prematuridade prévia [não (referência) $/ \operatorname{sim}$ (risco), $\beta=0,24$ ], uso de medicamentos [não (referência)/sim (risco), $\beta=-0,24$ ], tabagismo atual [não (referência) $/ \operatorname{sim}$ (risco), $\beta=0,24$ ], idade em que começou a fumar $(\beta=-0,02)$, tempo de fumo $(\beta=0,01)$, etilismo atual [não (referência)/sim (risco), $\beta=0,22]$ e níveis de hemoglobina $(\beta=-0,03)$, de acordo com a Tabela 4 .

Após controle para os fatores de confusão pela análise de regressão linear múltipla, as seguintes variáveis permaneceram associadas ao consumo de cafeína no modelo final: idade na menarca $(\beta=-0,15$; IC95\% $=-0,10--0,007$; $\mathrm{p}=0,02)$, número de pessoas que vivem na casa $(\beta=0,17$; IC95\% $=0,01-0,12 ; \mathrm{p}=0,01$ ) e uso de medicamentos [não (referência) $/ \operatorname{sim}$ (risco), $\beta=-0,24$; IC95\% $=-0,52--0,15$; $\mathrm{p}=<0,001]$. Esse modelo respondeu por $10,1 \%$ ( $\mathrm{R}^{2}$ ajustado) da variabilidade observada no consumo de cafeína das gestantes que pode ser, possivelmente, atribuída aos fatores de risco estudados (dados não apresentados em tabela).

\section{Discussão}

O consumo de produtos cafeinados durante o período gestacional tem sido muito estudado, devido à preocupação com as conseqüências que tal ingestão possa acarretar, tanto para a gestante quanto para o concepto, como o baixo peso ao nascer e a prematuridade. No entanto, poucos estudos têm atentado para os fatores que influenciam no consumo de cafeína durante este período.

No presente trabalho, verificou-se que a prevalência do consumo de produtos cafeinados em gestantes foi de 96\%. Este dado foi muito próximo ao encontrado em mulheres grávidas $(95 \%)^{22}$ e em estudo transversal com 4.757 homens e mulheres de várias idades, na Austrália, dos quais $97 \%$ eram consumidores de cafeína ${ }^{23}$. Em estudo realizado no Sul do Brasil foram encontradas prevalências de consumo de 93 e $92 \%$, respectivamente, entre casos (crianças com peso ao nascer menor que $2.500 \mathrm{~g}$ e idade gestacional maior que 28 semanas) e controles (próximas duas crianças nascidas no mesmo hospital que o caso, independente do seu peso ao nascer) durante a gestaçãa ${ }^{17}$.

Já em estudo realizado na Dinamarca, com amostra composta por 18.478 gestantes, a prevalência de consumo de cafeína encontrada foi de $57 \%{ }^{24}$. Essa porcentagem, quando comparada à do presente estudo, foi menor, provavelmente devido às características genéticas, culturais e de hábito alimentar, as quais variam entre as gestantes de cada país ou região. Os nossos resultados divergem também do observado no estudo realizado no Sul do Brasil ${ }^{17}$.

O café foi o alimento que mais contribuiu $(67,8 \%)$ para o consumo total de cafeína pelas gestantes e o com maior conteúdo médio da substância $(82,1 \mathrm{mg})$. Tal resultado confirma os resultados de outros estudos brasileiros - um realizado na cidade do Rio de Janeiro ${ }^{25}$ e o outro em Campinas, São Paulo ${ }^{1}$, nos quais o café foi também o mais consumido. Entretanto, no presente estudo, o alimento fonte de cafeína mais consumido pelas gestantes foi o refrigerante $(75,4 \%)$, assim como nos estudos conduzidos nos Estados Unidos, um analisando a dieta dos indivíduos ${ }^{26}$ e outro baseado nos dados do segundo National Health and Nutrition Examination Survey (NHANES II) ${ }^{27}$.

O consumo médio diário de cafeína, no presente estudo, foi de $121,1 \mathrm{mg}$. No estudo que avaliou a dieta de indivíduos americanos ${ }^{26}$, considerando-se apenas as gestantes da amostra, a média de consumo variou de 125 a $193 \mathrm{mg} / \mathrm{dia}$. O consumo médio calculado para as gestantes do estudo de Vlajinac et al. ${ }^{5}$ foi de 133,44 mg/dia e, portanto, também próximo ao valor obtido nesse estudo. Já em trabalho norte-americano com amostra representativa da população entre nove e 80 anos de idade ${ }^{28}$, incluindo gestantes, o consumo de cafeína foi, em média, duas a três vezes inferior ao relatado no estudo que avaliou a dieta de indivíduos americanos ${ }^{26}$ e ficando em torno de $58 \mathrm{mg} /$ dia no presente trabalho.

Estas discrepâncias ocorrem, pois o valor médio de cafeína varia de acordo com o conteúdo da substância nas fontes avaliadas pelos estudos, com o modo de preparo e com o tipo e a marca dos produtos utilizados ${ }^{1,5,6,17,25}$, além de ser influenciado pelas variações entre as populações, de indivíduo para indivíduo, e até quando a mesma pessoa prepara o produto em condições aparentemente idênticas e no mesmo dia ${ }^{9,18}$.

Estudo realizado com gestantes de Connecticut e Massachusetts9 revelou que as seguintes variáveis estão 
associadas ao consumo elevado de cafeína: idade materna menor ou igual a 24 anos, maior número de gestações e partos, ser casada, ser hispânica, baixa escolaridade, fumar dez ou mais cigarros por dia durante o primeiro e o terceiro trimestres de gestação e consumo de álcool superior a $28,3 \mathrm{~g}$ por dia durante o primeiro trimestre.

Para os autores do estudo realizado no Sul do Brasil ${ }^{17}$, grandes consumidoras de cafeína $(\geq 300 \mathrm{mg} / \mathrm{dia}$ ao longo da gestação), em geral, viviam em áreas urbanas, eram brancas, magras e altas, e apresentavam menores IMC. Elas também estavam mais propensas a fumar, consumir bebida alcoólica, ter menos relações sexuais no último mês da gestação e comparecer menos freqüentemente ao pré-natal. Já no presente estudo, observou-se, na análise de regressão linear múltipla, que o consumo de cafeína mostrou associação com a idade na menarca, número de pessoas na casa e uso de medicamentos.

Em relação ao modelo final de regressão, quanto maior a idade na menarca, menor foi o consumo de cafeína pelas gestantes. Ou seja, gestantes cuja menarca foi precoce consumiriam mais cafeína que as gestantes com menarca tardia. Sabe-se que a idade precoce na menarca é fator de risco para osteoporose e estaria também associada ao câncer de mama $^{29}$, não havendo na literatura nenhuma relação fundamentada entre consumo de cafeína e idade na menarca. Fato é que as mulheres que apresentam a menarca mais cedo estão submetidas à ação antecipada do estrogênio ${ }^{29}$ que, juntamente com a progesterona, apresenta um efeito antagonista no metabolismo da cafeína, provavelmente por indução de enzimas microssomais hepáticas, ocasionando aumento da concentração de cafeína plasmática ${ }^{16}$. Assim sendo, deve-se atentar para o nível de ingestão diária dessas mulheres, pois a concentração plasmática de cafeína aumentaria ainda mais com o consumo excessivo dessa metilxantina.

O consumo de cafeína foi maior entre as gestantes que moravam com mais pessoas. Uma possível justificativa para tal achado seria o fato de que o maior número de moradores em casa estivesse relacionado ao maior número de filhos e à presença do companheiro em casa, o que, inevitavelmente, significaria maior gasto com a alimentação e, conseqüentemente, indicaria a opção por produtos mais baratos e que rendam maior quantidade - caso do café, refrigerantes e chás; logo, maior consumo de produtos cafeinados. Tal associação foi observada indiretamente pelo estudo realizado com gestantes de Connecticut e Massachusetts ${ }^{9}$, no qual as mulheres com maior número de gestações e partos e casadas apresentaram maior consumo de cafeína durante o primeiro trimestre gestacional.

Este estudo demonstrou que gestantes que não faziam uso de medicamentos tinham um consumo maior de cafeína. Os medicamentos mais consumidos neste início da gestação foram suplementos vitamínicos e remédios para dores e enjôos. O maior consumo de cafeína entre as gestantes que não utilizavam esses remédios pode ser atribuído ao fato de serem essas mulheres as que comparecem menos freqüentemente ao pré-natal ${ }^{17}$ e que, portanto, não receberiam orientação para utilização de algum medicamento nem para limitarem o consumo de cafeína durante a gestação, prática comum e recomendada nos atendimentos pré-natais. De acordo com estudo realizado no Sul do $\mathrm{Brasil}^{17}$, grandes consumidoras de cafeína compareceram menos freqüentemente ao pré-natal e, segundo estudo realizado em Viena ${ }^{12}$, o maior consumo de cafeína esteve associado a um estilo de vida menos saudável. Logo, deve-se procurar, a partir da identificação dessas gestantes, inseri-las no atendimento pré-natal e orientá-las quanto aos benefícios deste e de um estilo de vida mais saudável.

Em relação ao tabagismo, 11,4\% das gestantes relataram fumar, enquanto $25,1 \%$ eram ex-tabagistas. A relação entre o consumo da cafeína na gestação e o fumo é complexa. $\mathrm{O}$ atual estudo não confirmou os resultados obtidos nos estudos realizados com gestantes de Connecticut e Massachusetts ${ }^{9}$, no Sul do Brasil ${ }^{17}$ e baseado nos dados do NHANES II $^{27}$, os quais demonstraram que gestantes fumantes têm um consumo aumentado de cafeína em relação às não fumantes. $\mathrm{O}$ estudo realizado com gestantes de Connecticut e Massachusetts ${ }^{9}$ revelou também que o consumo de cafeína aumenta conforme aumenta o número de cigarros fumados.

Observou-se um percentual de $12,6 \%$ de não respondentes na entrevista e no QFCA. O tipo de delineamento utilizado, bem como as variáveis do estudo do ponto de vista da plausibilidade temporal e biológica, não possibilitaram estabelecer uma relação temporal de causa e efeito (visto que as informações sobre exposição e desfecho foram obtidas ao mesmo tempo), mas permitiu que fossem exploradas algumas associações entre estas variáveis estudadas.

O estudo não coletou informações a respeito do consumo de chá e não quantificou a cafeína proveniente de medicamentos, apesar de questionar as mulheres a respeito dos medicamentos utilizados e saber que apenas quatro gestantes $(1,6 \%)$ informaram o uso de algum medicamento contendo cafeína. Quando a cafeína é a substância de interesse, sabe-se que quantificar apenas o consumo de café, mesmo sendo este o alimento fonte principal de cafeína, subestima o consumo dessa última. Assim, o ideal é quantificar os principais alimentos fontes, como café, chás e refrigerantes ${ }^{30}$. Neste aspecto, o presente artigo foi além, pois contabilizou o consumo de achocolatados, chocolates e mate. Ademais, procurou trabalhar com teores de cafeína calculados para produtos no Brasil, como foi o caso do achocolatado em pó, do chocolate em barra e do mate, com base nos achados de estudo realizado no 
Sul do país ${ }^{17}$, e do café, segundo dados do estudo cujas autoras analisaram o teor de cafeína em diversas marcas de cafés brasileiros ${ }^{18}$.

Conclui-se que o consumo de cafeína foi considerado baixo, apesar de freqüente. Uma minoria das gestantes relatou consumo superior ao limite de $300 \mathrm{mg} / \mathrm{dia}^{1,9,17}$ atualmente preconizado. Por outro lado, observou-se tendência a este consumo elevado em determinado grupo de gestantes. Influenciou bastante a falta de estudos acerca do tema, que permitissem melhor comparabilidade com os achados aqui encontrados.

Do ponto de vista nutricional, ressaltam-se alguns hábitos não saudáveis, como o consumo elevado de refrigerantes e o consumo dos próprios alimentos fontes de cafeína, a partir do momento que prejudiquem a biodisponibilidade de nutrientes importantes na dieta das gestantes avaliadas. A qualidade nutricional da alimentação deve ser muito bem avaliada durante a gestação, procurando, no caso deste estudo, identificar as gestantes propensas ao consumo elevado de cafeína e melhor orientá-las nutricionalmente durante o atendimento pré-natal prestado.

\section{Colaboradores}

Alice Helena de Resende Nóra Pacheco participou de todas as etapas do estudo, desde o planejamento, coleta de dados, levantamento bibliográfico, análise dos resultados e elaboração do artigo. Daniele Marano Rocha Araujo contribuiu na coleta de dados, análise dos resultados e revisão do artigo. Elisa Maria de Aquino Lacerda colaborou na revisão metodológica, na quantificação do consumo de cafeína e na revisão do artigo. Gilberto Kac participou do planejamento da coorte, análise dos resultados, orientação e revisão do artigo.
1. Bicalho GG, Barros Filho AA. Peso ao nascer e influência do consumo de cafeína. Rev Saúde Pública. 2002;36(2):180-7.

2. Grosso LM, Bracken MB. Caffeine metabolism, genetics, and perinatal outcomes: a review of exposure assessment considerations during pregnancy. Ann Epidemiol. 2005; 15(6):460-6.

3. Barone JJ, Roberts HR. Caffeine consumption. Food Chem Toxicol. 1996;34(1): 119-29.

4. Rifas-Shiman SL, Rich-Edwards JW, Willett WC, Kleinman KP, Oken E, Gillman MW. Changes in dietary intake from the first to the second trimester of pregnancy. Paediatr Perinat Epidemiol. 2006;20(1):35-42.

5. Vlajinac HD, Petrović RR, Marinković JM, Sipetić SB, Adanja BJ. Effect of caffeine intake during pregnancy on birth weight. Am J Epidemiol. 1997; 145(4):335-8.

6. Cook DG, Peacock JL, Feyerabend C, Carey IM, Jarvis M, Anderson HR, et al. Relation of caffeine intake and blood caffeine concentrations during pregnancy to fetal growth: prospective population based study. BM. 1996;313(7069):1358-62.

7. Rondó PH, Rodrigues LC, Tomkins AM. Coffee consumption and intrauterine growth retardation in Brazil. Eur J Clin Nutr. 1996;50(1 1):705-9.

8. Institute of Medicine. Nutrition during pregnancy. Part I: weight gain, Part II: nutrient supplements. Washington: National Academy Press; 1990

9. Bracken MB, Triche EW, Belanger K, Hellenbrand K, Leaderer BP. Association of maternal caffeine consumption with decrements in fetal growth. Am J Epidemiol. 2003;157(5):456-66.

10. Bech BH, Obel C, Henriksen TB, Olsen J. Effect of reducing caffeine intake on birth weight and length of gestation: randomised controlled trial. BM. 2007;334(7590):409.Comment in: BM. 2007;334(7590):377; Republished in: Ugeskr Laeger. 2007; 169(39):3300-2.

11. Higdon JV, Frei B. Coffee and health: a review of recent human research. Crit Rev Food Sci Nutr. 2006;46(2):101-23.
12. Schwarz B, Bischof HP, Kunze M. Coffee, tea, and lifestyle. Prev Med. 1994;23(3):377-84.

13. Godzik B, Manning WJ, Sichieri R, Everhart JE. Validity of a Brazilian food frequency questionnaire against dietary recalls and estimated energy intake. Nutr Res. 1998;18(10):1649-59.

14. Pinheiro ABV, Lacerda EMA, Benzecky EG, Gomes MCS, Costa VM. Tabela para avaliação de consumo alimentar em medidas caseiras. $5^{a}$ ed. São Paulo: Athenev; 2004.

15. United States Department of Agriculture. Agricultural Research Service. USDA National Nutrient Database for Standard Reference, Release 18 [Internet]. 2005 [cited 2007 abr 9]. Available from: http://www.nal.usda.gov/fnic/foodcomp/search/

16. World Health Organization. International Agency for Research on Cancer. IARC Monographs on the Evaluation of Carcinogenic Risks to Humans. Coffee, tea, mate, methylxanthines and methylglyoxal. Lyon: International Agency for Research on Cancer; 1991. v. 51.

17. Santos IS, Victora CG, Huttly S, Carvalhal JB. Caffeine intake and low birth weight: a population-based case-control study. Am J Epidemiol. 1998;147(7):620-7.

18. Camargo MCR, Toledo MCF. Teor de cafeína em cafés brasileiros. Ciênc Tecnol Alimentos. 1998;18(4):421-4.

19. Lohman TG, Roche AF, Martorell R. Anthropometric standadization reference manual. Champaign: Human Kinetics Books; 1988.

20. Mondini L, Levy RB, Saldiva SRDM, Venâncio SI, Aguiar JA, Stefanini MLR. Prevalência de sobrepeso e fatores associados em crianças ingressantes no ensino fundamental em um município da região metropolitana de São Paulo, Brasil. Cad Saúde Pública. 2007;23(8): 1825-34.

21. Conselho Nacional de Saúde. Resolução n $196 / 96$ sobre pesquisa envolvendo seres humanos. Bioética. 1996;4(2 Supl):15-25.

22. Watkinson B, Fried PA. Maternal caffeine use before, during and after pregnancy and effects upon offspring. Neurobehav Toxicol Teratol. $1985 ; 7(1): 9-17$ 
23. Shirlow M. Patterns of caffeine consumption. Hum Nutr Appl Nutr. 1983;37(4):307-13.

24. Wisborg K, Kesmodel U, Bech BH, Hedegaard M, Henriksen TB. Maternal consumption of coffee during pregnancy and stillbirth and infant death in first year of life: prospective study. BM. 2003;326(7386):420-3.

25. Souza RAG, Sichieri R. Consumo de cafeína e de alimentos - fonte de cafeína e prematuridade: um estudo caso - controle. Cad Saúde Pública. 2005;21(6):1919-28.

26. Frary $C D$, Johnson RK, Wang $M Q$. Food sources and intakes of caffeine in the diets of persons in the United States. J Am Diet Assoc. 2005;105(1):110-3.
27. Klesges RC, Ray JW, Klesges LM. Caffeinated coffee and tea intake and its relationship to cigarette smoking: an analysis of the Second National Health and Nutrition Examination Survey (NHANES II). J Subst Abuse. 1994;6(4):407-18.

28. Knight CA, Knight I, Mitchell DC, Zepp JE. Beverage caffeine intake in US consumers and subpopulations of interest: estimates from the Share of Intake Panel survey. Food Chem Toxicol. 2004;42(12):1923-30.

29. Vieira JGH, Brandão CMA. Medindo a densidade mineral óssea e algo mais. Arq Bras Endocrinol Metab. 1998;42(5):331-2.

30. Brown J, Kreiger N, Darlington GA, Sloan M. Misclassification of exposure: coffee as a surrogate for caffeine intake. Am J Epidemiol. $2001 ; 153(8): 815-20$. 